\title{
Étude théorique et expérimentale du comportement non-linéaire d'un engrenage droit induit par le jeu entre dents
}

\author{
Sébastien Espin ${ }^{1}$, Jö̈l Perret-Liaudet ${ }^{1, a}$ et Ahmet Kahraman ${ }^{2}$ \\ 1 Laboratoire de Tribologie et Dynamiques des Systèmes, UMR 5513, École Centrale de Lyon, 69134 Écully Cedex, France \\ 2 Department of Mechanical Engineering, The Ohio State University, Columbus, OH 43210-4678, USA
}

Reçu le 23 novembre 2006, accepté le 29 mai 2007

\begin{abstract}
Résumé - Nous nous intéressons dans cette étude à un modèle dynamique non-linéaire d'un engrenage droit à un degré de liberté. Ce modèle permet de prendre en compte la présence du jeu entre dents ainsi que la non-linéarité de la raideur d'engrènement associée à l'évolution instantanée du nombre de dents en prise. Notre étude concerne le cas d'engrenages corrigés par des corrections linéaires en tête de denture ainsi que des engrenages non corrigés. Le but de cette étude a été d'analyser la pertinence de ce modèle vis-à-vis de résultats expérimentaux mettant en œuvre un banc d'essais dont les caractéristiques, notamment aux niveaux de la micro-géométrie des dentures, sont parfaitement contrôlées. En conclusion, les résultats comparatifs sont encourageants, même si nous n'avons pas pu reproduire par simulation certains phénomènes non-linéaires observés. Parmi ceux-ci, on peut citer le cas de la cascade harmonique observée sur un engrenage corrigé et prenant naissance au niveau de la résonance principale.
\end{abstract}

Mots clés : Engrenage / raideur d'engrènement / jeu / vibration / non-linéaire / impact / modèle / expérimental

\begin{abstract}
Theoretical and experimental study of the nonlinear behaviour of a spur gear pair induced by tooth backlash. We are interesting in this study by a non-linear dynamic modelling of spur gears. This model allows to take into account the tooth backlash and also the non-linearity of the meshing stiffness associated to the instantaneous change of the number of teeth in contact. Our study concerns spur gears possessing, or not, modifications in form of linear tip relief. The goal was to analyse the ability of the introduced modelling for reproducing experimental results. These experimental results were obtained on a convenient test device for which characteristics, in particular the micro geometry of teeth, are perfectly controlled. To conclude, comparative results are encouraging even if several experimental non-linear phenomena are not reproduced by numerical simulations. Among these, one can cite the case of the period doubling route which takes place, for the analysed corrected gear, on the primary resonance.
\end{abstract}

Key words: Gear / meshing stiffness / backlash / vibration / non-linear / impact / modelling / experimental

\section{Introduction}

Le comportement dynamique des transmissions par engrenages résulte principalement, si l'on ne retient que les sources d'excitation interne, de l'erreur statique de transmission [1-5]. Cette erreur est généralement définie comme l'écart entre la position angulaire qu'occupe la roue de sortie et celle qu'elle devrait occuper si l'engrenage était parfait et infiniment rigide [5]. Les sources physiques à cette erreur sont d'une part les écarts de

\footnotetext{
a Auteur correspondant :

joel.perret-liaudet@ec-lyon.fr
}

géométrie entre le flanc théorique de la denture et le flanc réel, et d'autre part les déformations élastiques notamment celles associées à la denture correspondant à ce que l'on nomme la raideur d'engrènement. Notons pour cette dernière que sa nature paramétrique (fluctuation périodique de ses caractéristiques) est déterminante sur le comportement dynamique de la transmission. Sous certaines conditions, l'erreur statique de transmission peut exciter l'engrenage de telle sorte qu'apparaissent des pertes de contact entre denture. Ces pertes de contact sont possibles compte tenu de la présence d'un jeu entre dents nécessaire notamment pour le montage de 


\section{Nomenclature}

\begin{tabular}{|ll|}
\hline Lettres latines & \\
$c$ & amortissement visqueux équivalent (viscance) \\
$e$ & excitation déplacement associé aux défauts de géométrie \\
$E$ & déformation d'un couple de dents en prise sous charge statique \\
$f$ & force élastique non-linéaire de denture adimensionnelle \\
$F$ & force élastique non-linéaire de denture \\
$h$ & dépouille de denture \\
$I$ & inertie en rotation des pignons \\
$k$ & raideur de contact pour un couple de dents en prise \\
$K$ & raideur d'engrènement \\
$m$ & masse équivalente de l'engrenage \\
$n$ & nombre théorique de dents en prise \\
$N$ & force statique transmise \\
$q$ & erreur dynamique de transmission adimensionnelle \\
$R$ & rayon de base des pignons \\
$t$ & temps \\
$x$ & erreur dynamique de transmission \\
Lettres grecques & \\
$\delta$ & déformation élastique \\
$\varepsilon_{\alpha}$ & rapport de conduite \\
$\zeta$ & taux d'amortissement visqueux équivalent \\
$\theta$ & rotation des pignons \\
$\lambda$ & rapport entre la raideur de contact et la raideur moyenne d'engrènement \\
$\sigma$ & force statique adimensionnelle \\
$\omega$ & temps adimensionnel \\
\hline & pulsation d'engrènement adimensionnelle \\
\hline & \\
\hline & \\
\hline &
\end{tabular}

l'engrenage. Ces pertes de contact se caractérisent par des phénomènes de vibro-impact, pouvant conduire à des surcharges dynamiques importantes, des dégradations et de forts niveaux sonores.

Dans l'état des connaissances actuelles, des efforts restent à faire au niveau des modèles prédictifs de ce comportement dynamique non-linéaire. L'étude théorique de ce comportement, vu la complexité des phénomènes non-linéaires mis en jeu, s'appuie généralement sur des modèles à faibles nombres de degré de liberté, typiquement un seul que constitue l'erreur dynamique de transmission [6-20]. L'ensemble des modèles proposés se distingue en trois groupes principaux du point de vue de la prise en compte des actions de contact au niveau de l'engrènement. Dans un premier groupe, seule la perte de contact est prise en compte comme source non-linéaire $[6,7]$. La raideur d'engrènement est dans ce cas introduite uniquement via sa valeur moyenne. Dans un second groupe et au contraire du précédent, elle est introduite comme une fonction périodique du temps [8-16]. Cette dépendance temporelle résulte principalement de la fluctuation périodique du nombre de dents en prise au cours de l'engrènement. Pour ces deux premiers groupes, la raideur d'engrènement (ou disons de contact) est essentiellement linéaire. Dans le cas du troisième groupe de modèles, la nature non-linéaire de la raideur d'engrènement est cette fois-ci introduite [17-20]. Ainsi la raideur d'engrènement est évaluée à partir de la réponse dynamique instantanée. Ces modèles permettent entre autres de prendre en compte les pertes partielles de contact sur un seul couple de dents en prise.

Du point de vue expérimental, plusieurs études ont clairement mis en évidence le comportement non-linéaire induit par la présence du jeu entre dents, notamment dans le cadre des engrenages à denture droite [13, 14, 21-23]. Les comparaisons entre ces données expérimentales et les simulations numériques entreprises à ce jour ont essentiellement concerné les modèles des deux premiers groupes. Très peu de comparaisons avec des modèles du troisième groupe ont été réalisées et c'est précisément l'objectif de cette étude que d'apporter quelques éléments à ce sujet. Pour ce faire, nous avons choisi de comparer des résultats expérimentaux à des prédictions réalisées à partir d'un modèle décrit dans [20,24]. Dans cet article, la section 2 est consacrée à la description du montage expérimental utilisé. Dans la section 3, nous nous attachons à décrire le modèle dynamique introduit pour les simulations numériques. Enfin, les résultats numériques et expérimentaux sont présentés section 4. 


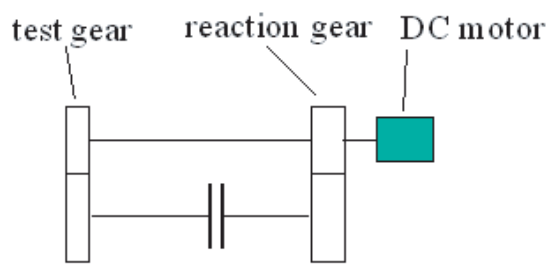

Fig. 1. Schéma de principe.

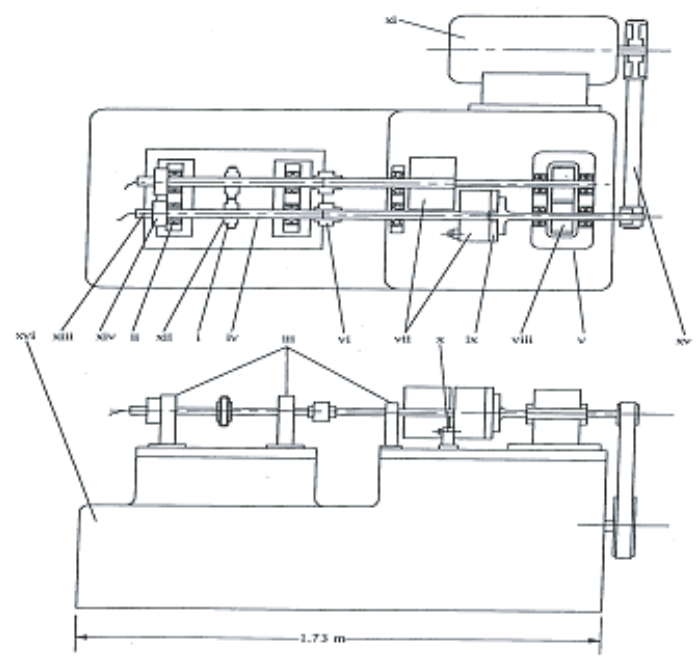

Fig. 2. Description du banc d'essais : engrenage d'essais (i), roulements sphériques (ii), supports des roulements (iii), arbres (iv), réducteur esclave (v), accouplements flexibles (vi), volants d'inertie (vii), engrenage hélicoïdal (viii), limiteur de couple (ix), moteur CC (xi), accéléromètres piézoélectriques (xii), courroie d'entraînement (xv), bâti (xvi).

\section{Montage expérimental}

Afin d'étudier le comportement dynamique nonlinéaire d'engrenages droits, nous avons exploité un banc d'essais de type circulation de puissance installé à l'Ohio State University (USA). Un schéma de principe est présenté figure 1 et la description du banc, figure 2 .

Ce banc d'essais est constitué de deux paires d'engrenages, l'une associée aux pignons étudiés et l'autre permettant une reprise d'effort. Ce type de montage permet d'assurer une régulation du couple avec précision et un fonctionnement stationnaire (vitesse régulière). L'engrenage d'essais (i) est monté sur paliers sphériques (ii) qui sont supportés par des supports rigides (iii). Les arbres (iv) ont des caractéristiques mécaniques permettant d'assurer un comportement dynamique purement de torsion. La paire de pignons testée est isolée d'un point de vue dynamique de la transmission (boîte réactive ou esclave) permettant la reprise des efforts (v) à l'aide d'accouplements à joints élastomères (vi) et de volants d'inerties (vii). La boîte réactive est constituée d'engrenages hélicoïdaux de précision (viii) dont les nombres de dents sont tels que les fréquences d'engrènements sont distinctes de celles de la boîte d'essais. Le couple appliqué est

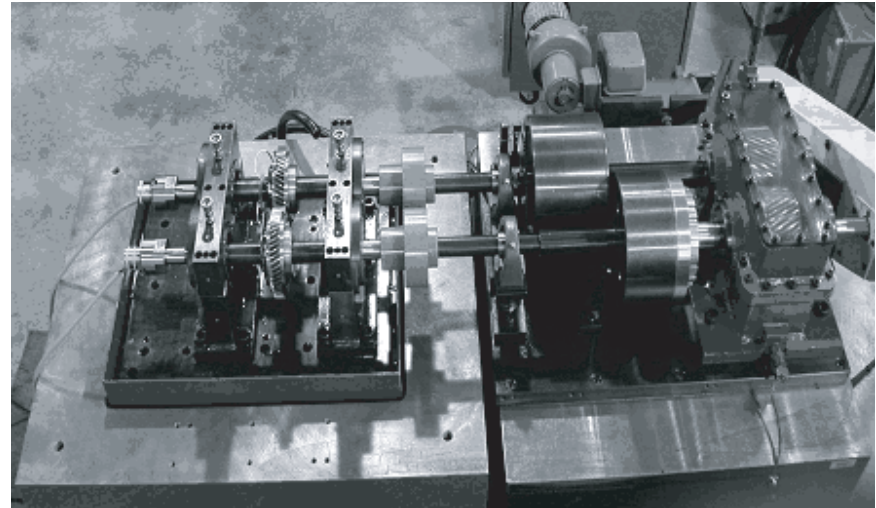

Fig. 3. Photo du montage expérimental.

ajusté en jouant sur un limiteur de couple. La puissance est fournie par un moteur à courant continu (xi) asservi en vitesse. La gamme de vitesses pour le banc est comprise entre 0 et 4500 tr.min ${ }^{-1}$. L'entraînement est assuré par un système poulie courroie lisse (xv). La lubrification s'effectue à l'aide d'une pompe (Dextron-II) à température et débit contrôlé afin de garantir un taux d'amortissement à peu près constant. Des mesures précédentes ont montré que ce taux se situait autour de $2 \%$. Enfin, le banc d'essais est monté sur un massif rigide (xvi), le marbre de la boîte d'essais étant découplé de celui de la boîte réactive.

La fabrication et le montage des bancs ont été réalisés avec une haute précision : ils correspondent à une classe de qualité 2 ou 3 par rapport au standard AFNOR. À titre d'exemple, les logements des roulements ont été réalisés in situ pour chaque jeu d'arbres et d'engrenages. La figure 3 présente une photographie du montage expérimental. Le banc d'essais est instrumenté de telle sorte que l'on puisse mesurer l'Erreur Dynamique de Transmission. Par ailleurs, on contrôle la vitesse de fonctionnement, le couple transmis et la température. Deux montages permettent la mesure de cette erreur. Le premier est constitué de codeur optique (xiii) permettant de travailler en basse fréquence (faible régime de vitesse) et le deuxième est constitué d'accéléromètres piézoélectriques (xii) montés sur chacune des roues dentées. Au cours des essais que nous avons réalisés, nous n'avons utilisé que le système accélérométrique.

Ce dernier est constitué pour chaque roue de quatre accéléromètres appareillés et montés comme décrit figure 4. Afin de mesurer l'accélération angulaire de chacun des pignons, les signaux des quatre accéléromètres sont additionnés par un boîtier analogique. Ainsi, la gravité et les éventuels mouvements transversaux sont éliminés. L'accélération angulaire résultante est filtrée par un filtre passe haut puis intégrée deux fois afin d'accéder aux déplacements. Le dispositif complet permet une résolution de $0,01 \mu \mathrm{m}$ avec une précision de $\pm 0,3 \mu \mathrm{m}$ et ce sur une bande de fréquences comprises entre 500 et $5000 \mathrm{~Hz}$. La répétitivité des mesures s'est avérée inférieure à $1 \mu \mathrm{m}$ sur des périodes d'essais supérieures à 3 ans incluant plus d'une centaine de tests. L'analyse des signaux s'est appuyée sur la mise en œuvre d'un analyseur 

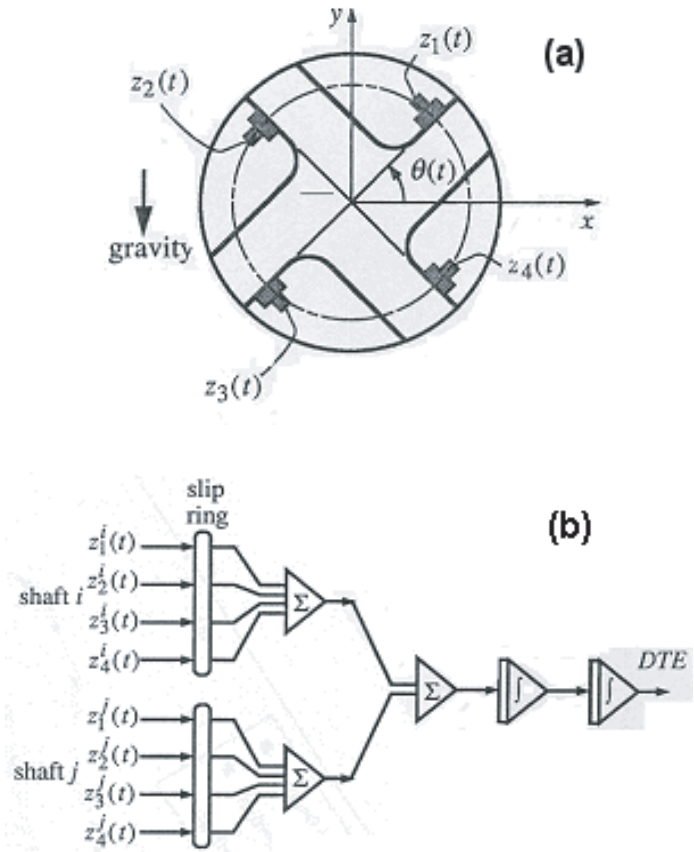

Fig. 4. Système de mesure de l'erreur dynamique de transmission : (a) montage des 4 accéléromètres sur les roues; (b) intégration analogique permettant d'obtenir l'erreur dynamique de transmission.

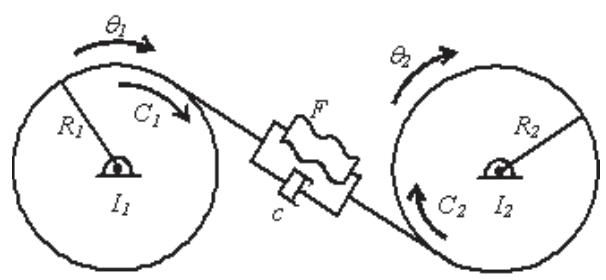

Fig. 5. Modèle dynamique.

spectral classique. La valeur rms des signaux présentés a été évaluée quant à elle en considérant les six premiers harmoniques de la demi-fréquence d'engrènement, soit :

$$
x_{\mathrm{rms}}^{2}=\sum_{p=1}^{3}\left(x_{p-\frac{1}{2}}^{2}+x_{p}^{2}\right)
$$

où $x$ constitue l'erreur dynamique de transmission et $p$ l'ordre de l'harmonique de la fréquence d'engrènement.

\section{Modèle dynamique}

\section{1 Équation du mouvement}

Afin de simuler le comportement non-linéaire de l'engrenage, on considère un modèle simplifié à deux degrés de liberté que sont les rotations des deux pignons en prise $\theta_{1}$ et $\theta_{2}$. Ce modèle est illustré figure 5 . Il est constitué de deux disques rigides dont les inerties polaires, pour le pignon menant et le pignon mené, sont notées respectivement $I_{1}$ et $I_{2}$. La seule composante élastique introduite dans le modèle correspond au couplage induit par le processus d'engrènement. La force de rappel élastique nonlinéaire $F$ qui en résulte est par hypothèse portée par la ligne d'action théorique. Les rayons de base sont notés $R_{1}$ et $R_{2}$. Un amortissement visqueux équivalent linéaire (coefficient $c$ ) est introduit afin de prendre en compte les effets dissipatifs.

En éliminant le mode de corps rigide (système élastique semi-défini positif), et en introduisant l'Erreur Dynamique de Transmission $x$, exprimée le long de la ligne d'action théorique selon :

$$
x=R_{1} \theta_{1}+R_{2} \theta_{2}
$$

le système élastique se réduit à un système non-linéaire à un seul degré de liberté dont l'équation du mouvement s'écrit :

$$
m \ddot{x}+c \dot{x}+F(x, e, t)=N
$$

où $m$ constitue une masse équivalente déduite des rayons de base et des inerties en rotation, et $N$ est la force statique transmise exprimée sur la ligne d'action théorique. Par ailleurs, $F(x, e, t)$ représente formellement la contribution élastique de l'effort dynamique de denture qui dépend de la raideur d'engrènement mais aussi des corrections et défauts de géométrie des dentures représentés par la fonction $e(t)$. Les caractéristiques essentielles de $F(x, e, t)$ sont sa nature paramétrique associée à la fluctuation de la raideur d'engrènement, d'une part et d'autre part, sa nature non-linéaire associée aux pertes de contact. La plupart des modèles considèrent soit l'une ou l'autre de ces caractéristiques, soit dissocient l'effet paramétrique de la perte de contact. Dans cette étude, on se propose d'introduire un modèle pour lequel les conditions du contact évoluent de manière indépendante pour les deux paires de dents en prise théorique [20,24]. Par contre, la raideur de contact pour un couple de dents en prise, notée $k$ ci-après, est supposée linéaire et constante. Finalement, l'expression de la composante élastique de l'effort dynamique de denture s'exprime :

$$
F=\sum_{i=1}^{n(t)} k \delta_{i} H\left(\delta_{i}\right)
$$

Dans cette équation, la fonction $n(t)$ constitue le nombre de paires de dents pouvant être en prises à l'instant $t$ et $H($ ) est la fonction échelon unité d'Heaviside. Par ailleurs, $\delta_{i}$ représente la déformation élastique (lorsqu'elle est positive) associée à la paire de dents potentiellement en prise $\mathrm{n}^{\circ} i$. Il vient :

$$
\delta_{i}=x-e_{i}(t)
$$

où $e_{i}(t)$ est l'erreur composée de géométrie de la paire de dents $n^{\circ} i$. Notons que l'expression (4) de l'effort de denture se distingue des modèles non-linéaires et paramétriques usuels où la composante élastique s'écrirait :

$$
F=k(t) \delta H(\delta)
$$

Ainsi, la source paramétrique n'est plus portée par la raideur d'engrènement, mais par la fluctuation du nombre 
de dents $n(t)$ potentiellement en prise. D'autre part, le modèle introduit peut tenir compte des pertes de contact partielles sur un seul couple de dents.

\section{2 Équation adimensionnelle}

À partir de $\bar{K}$, la raideur moyenne d'engrènement évaluée à partir du calcul statique de l'erreur de transmission, on introduit la pulsation propre linéarisée du système $\Omega$ telle que

$$
\Omega^{2}=\frac{\bar{K}}{m}
$$

Cette pulsation constituera les lieux fréquentiels où prendront naissance les résonances non-linéaires. Introduisons par ailleurs les variables suivantes :

$$
\begin{aligned}
\lambda & =\frac{k}{\bar{K}}, \quad 2 \zeta=\frac{c}{m \Omega}, \quad f=\frac{F}{E \bar{K}}, \quad \sigma=\frac{N}{E \bar{K}}, \\
\tau & =\Omega t \text { et } q=\frac{x}{E}
\end{aligned}
$$

$E$ constitue une longueur caractéristique du problème, $\zeta$ est le taux d'amortissement visqueux équivalent. $f, \sigma$, $\tau$, et $q$ sont sans dimension et respectivement l'effort dynamique de denture, la force statique transmise, le temps et le déplacement. L'équation (3) peut alors s'écrire sous la forme adimensionnelle suivante :

$$
q^{\prime \prime}+2 \zeta q^{\prime}+f=\sigma
$$

Dans cette équation, ' représente la dérivée par rapport au temps adimensionnel $\tau$. Par ailleurs, la contribution élastique à l'effort dynamique de denture sans dimension s'écrit :

$$
f=\lambda \sum_{i=1}^{n(\tau)} \hat{\delta}_{i} H\left(\hat{\delta}_{i}\right)
$$

avec

$$
\hat{\delta}_{i}=q-\frac{e_{i}}{E}=q-\hat{e}_{i}
$$

Dans le cas où l'engrenage est non corrigé, on choisit pour la valeur de $E$ :

$$
E=\frac{N}{k}
$$

qui correspond à la déformation élastique d'un seul couple de dents en prise induite par la charge statique transmise $N$. Dans ce cas $\lambda$ et $\sigma$ sont égaux. De plus, on peut remarquer que le rapport $\lambda$ se déduit directement du rapport de conduite $\varepsilon_{\alpha}$ par :

$$
\lambda=\frac{1}{\varepsilon_{\alpha}}
$$

Par contre, dans le cas d'une correction en tête, il est intéressant d'introduire la valeur de la dépouille notée $h$. Dans ce cas, nous avons choisi cette valeur $h$ pour $E$.

\subsection{Résolution}

Afin de résoudre les équations du mouvement, nous avons utilisé d'une part un schéma d'intégration temporelle explicite de type différences centrées, et d'autre part une technique de calcul de type méthode de tir assortie d'une technique de continuation standard. La méthode de tir utilisée est dans le cas forcé équivalente à la méthode des sections de Poincaré $[25,26]$.

\section{Résultats}

\subsection{Pignons étudiés}

Pour l'ensemble des résultats présentés, les couples d'engrenages sont constitués de pignons droits dont les nombres de dents sont identiques et égaux à 50 dents (rapport de transmission 1). Pour toutes les dentures, le module est égal à $3 \mathrm{~mm}$, l'angle de pression $20^{\circ}$, la largeur de denture $20 \mathrm{~mm}$ et l'entraxe $150 \mathrm{~mm}$. Au niveau des engrenages expérimentaux, l'erreur totale de division est inférieure à $10 \mu \mathrm{m}$ et les erreurs de profil et d'hélice inférieures à $3 \mu \mathrm{m}$. Enfin, une correction de bombé de $5 \mu \mathrm{m}$ concerne l'ensemble des dentures. Les différents cas présentés se distinguent par leur rapport de conduite, leur correction de profil, et la charge transmise. Dans le cas des dentures corrigées, il s'agit d'une correction linéaire sur chaque roue dont la dépouille est de $4 \mu \mathrm{m}$. La profondeur est telle que la dépouille évolue du cercle de tête à un peu moins que le cercle primitif (correction longue). Les tests sont réalisés pour une vitesse de rotation de 600 à 4000 tr.min ${ }^{-1}$. Cette plage de vitesse correspond à une fréquence d'engrènement comprise entre 500 et $3400 \mathrm{~Hz}$. Les fréquences propres linéarisées se situent pour les couples d'engrenages étudiés entre 2400 et $3200 \mathrm{~Hz}$.

\subsection{Denture non corrigée}

En premier lieu, nous nous sommes intéressés au cas de dentures non corrigées. Ce cas est intéressant car le modèle introduit équation (4) est équivalent au modèle non-linéaire et paramétrique plus classique décrit équation (6). On présente figure 6 un exemple typique de l'évolution de la réponse en fonction de la fréquence d'engrènement $\omega$ (adimensionnée par rapport à la fréquence propre linéarisée). Pour cet exemple, le rapport de conduite $\varepsilon_{\alpha}$ est de 1,75 . Pour la charge statique transmise $(340 \mathrm{Nm})$, la valeur de $E$ est de $20 \mu \mathrm{m}$.

En premier lieu, on constate sans surprise le caractère mollissant du système, l'ensemble des résonances nonlinéaires étant incliné vers des fréquences décroissantes. Ce caractère mollissant est à imputer à la non-linéarité de jeux. On reconnaît la résonance principale autour de $\omega=1$, ainsi que les 3 résonances sur harmoniques autour de $\omega=1 / p(p=2,3,4)$. On notera que l'effet de la non-linéarité est important puisque les résonances s'établissent sur de larges plages de fréquence, de l'ordre 


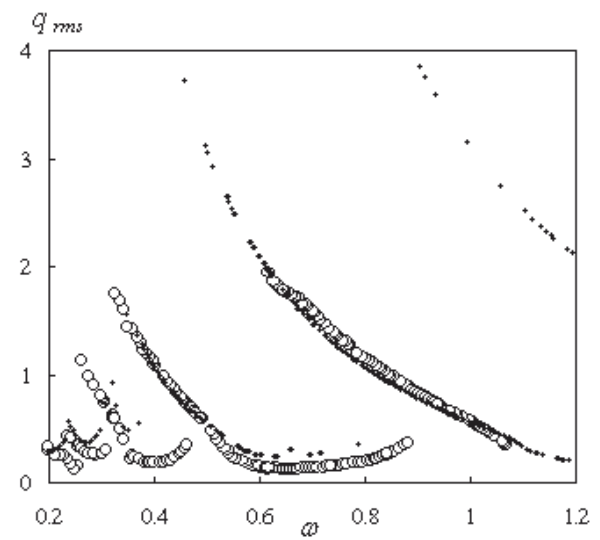

Fig. 6. Évolution de la réponse $\mathrm{rms}, q_{\mathrm{rms}}$, en fonction de la fréquence d'engrènement sans dimension $\omega$. Denture non corrigée, $\varepsilon_{\alpha}=1,75 ; E=20 \mu \mathrm{m}$. Résultats numériques obtenus par un balayage standard en fréquence $(\bullet)$ et résultats expérimentaux (०).

du simple au double. De plus, les niveaux atteints sont assez élevés, de l'ordre de $40 \mu \mathrm{m}$ pour une déformation moyenne de l'ordre de $10 \mu \mathrm{m}$. On constate par ailleurs que les résultats numériques sont en accord avec les résultats expérimentaux. Précisons que les simulations numériques ont été réalisées avec un taux d'amortissement visqueux équivalent de $3 \%$. Toutefois, les niveaux atteints aux pics de résonance sont plus élevés pour ce qui est des simulations. On peut avancer l'idée que, d'une part le taux d'amortissement réel équivalent est connu avec beaucoup d'incertitude en condition de comportement dynamique, et d'autre part que le bassin d'attraction pour la solution impactante diminue au fur et à mesure que l'amplitude de la réponse augmente (plus grande sensibilité aux perturbations extérieures ce qui peut conduire à un saut d'amplitude prématuré au cours des essais). On note par ailleurs la présence d'une branche pour les résultats numériques correspondant à la résonance sous harmonique principale initiée autour de $\omega=2$. On ne l'observe pas du point de vue expérimental, car il faudrait pour ce faire atteindre une vitesse de rotation de l'ordre de 8000 tr. $\mathrm{min}^{-1}$ pour l'accrocher. Enfin, les résultats numériques présentés ont été obtenus par des balayages standards en fréquence. Ils ne permettent donc pas de suivre fidèlement la solution 1-T périodique. À ce titre la méthode de tir assortie de la technique de continuation est plus adaptée. La figure 7 présente une comparaison des résultats obtenus par les deux méthodes numériques mises en œuvre. On constate un très bon accord entre les deux méthodes.

La figure 8 présente un deuxième exemple de résultats pour une denture non corrigée. Pour cet exemple, le rapport de conduite $\varepsilon_{\alpha}$ est de 1,1. Pour la charge statique transmise $(170 \mathrm{Nm})$, la valeur de $E$ est de $8 \mu \mathrm{m}$. On constate de nouveau le caractère mollissant du système. On reconnaît comme précédemment la résonance principale autour de $\omega=1$, ainsi que les 3 résonances sur

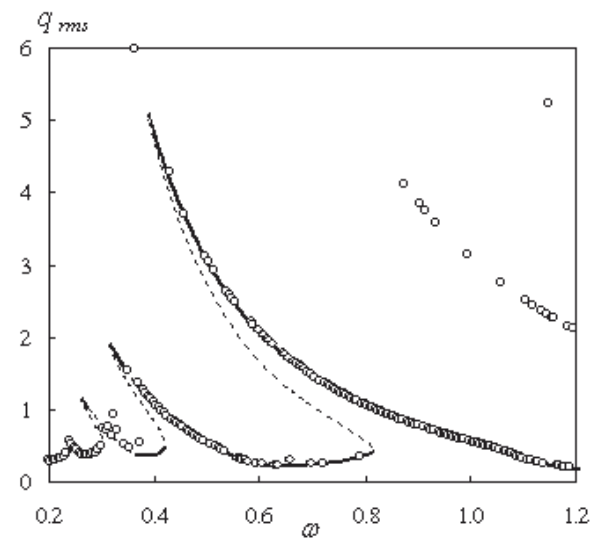

Fig. 7. Évolution de la réponse rms, $q_{\mathrm{rms}}$, en fonction de la fréquence d'engrènement sans dimension $\omega$. Denture non corrigée, $\varepsilon_{\alpha}=1,75 ; E=20 \mu \mathrm{m}$. Résultats numériques obtenus par un balayage standard (o) et par continuation : solutions 1T-périodiques stables (trait continu épais) et instables (trait fin discontinu).

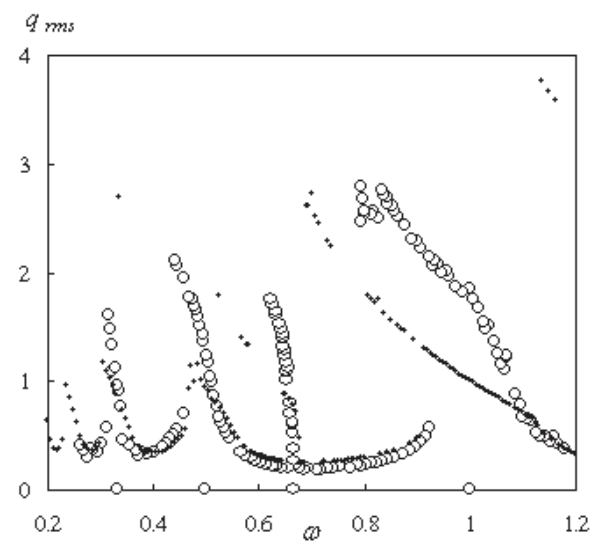

Fig. 8. Évolution de la réponse rms, $q_{\mathrm{rms}}$, en fonction de la fréquence d'engrènement sans dimension $\omega$. Denture non corrigée, $\varepsilon_{\alpha}=1,1 ; E=8 \mu \mathrm{m}$. Résultats numériques obtenus par un balayage standard en fréquence $(\bullet)$ et résultats expérimentaux (०).

harmoniques autour de $\omega=1 / p(p=2,3,4)$. Par ailleurs, on distingue nettement l'apparition d'une résonance de type sous harmonique d'ordre $p=2 / 3$ ( $0,66)$.

Plus précisément, les réponses observées sont de type 2T-périodiques dominées par l'harmonique d'ordre 3 (i.e. à la fréquence $3 / 2$ de la fréquence d'engrènement). Comme on peut le constater, les comparaisons entre simulations numériques et essais expérimentaux sont ici moins bonnes que dans le cas précédent. En particulier, la résonance principale s'incline de manière plus franche vers les basses fréquences dans le cas de la simulation. Par contre, l'apparition de la résonance $2 / 3$ sous harmonique est respectée. La figure 9 représente pour cette résonance un exemple de réponse temporelle observée pour $\omega$ légèrement inférieur à 0,66 . On constatera le bon accord entre prédiction et mesure. 

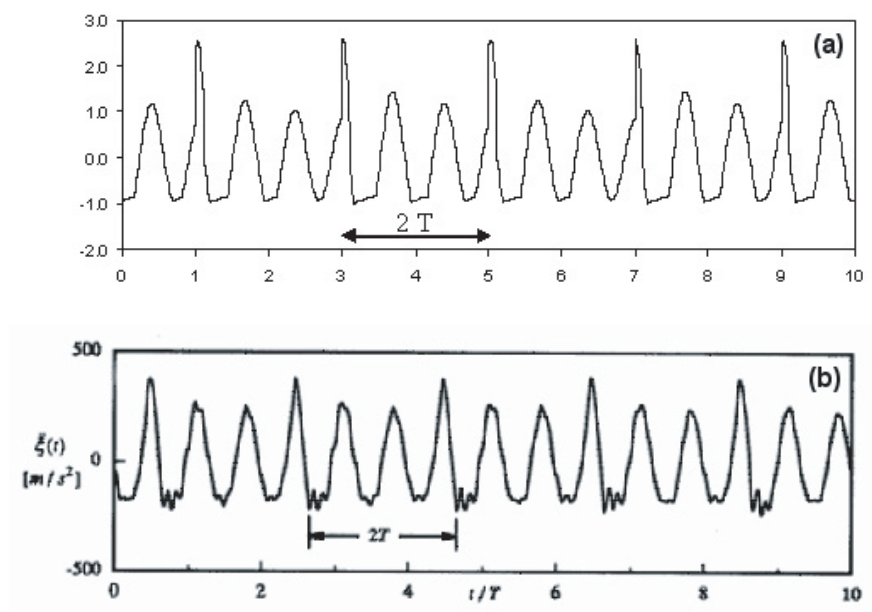

Fig. 9. Évolution temporelle de la réponse accélération pour $\omega$ légèrement inférieur à 1 . Denture non corrigée, $\varepsilon_{\alpha}=$ 1,$1 ; E=8 \mu \mathrm{m}$. Résultats numériques (a) et résultats expérimentaux (b).

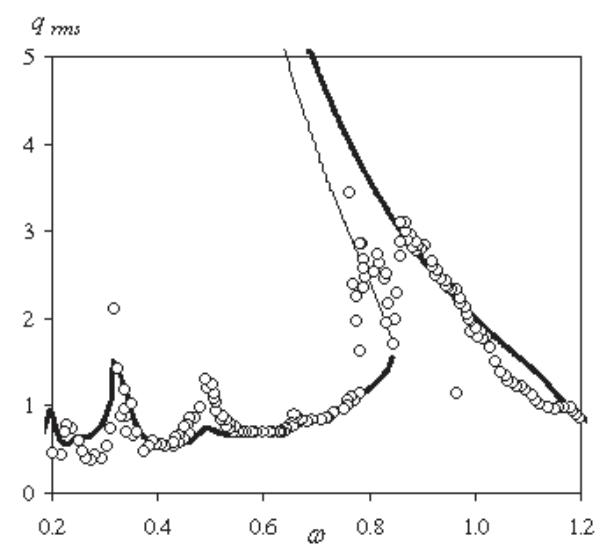

Fig. 10. Évolution de la réponse $\mathrm{rms}, q_{\mathrm{rms}}$, en fonction de la fréquence d'engrènement sans dimension $\omega$. Denture corrigée, $\varepsilon_{\alpha}=1,4 ; E=4 \mu \mathrm{m}$. Résultats numériques obtenus par continuation (stable : trait épais; instable : trait fin) et résultats expérimentaux (o).

\subsection{Denture corrigée}

La figure 10 montre un exemple de réponse pour une denture corrigée dont le rapport de conduite est de 1,4. Comme on peut le constater l'accord entre résultats numériques et expérimentaux est assez satisfaisant, sauf sur l'apparition de la cascade harmonique qui prend naissance sur la résonance principale.

En effet, les résultats expérimentaux obtenus pour $\omega$ compris entre 0,8 et 0,9 correspondent en fait à ce type de phénomènes. Nous n'avons pas pu dans le cas de figure de cet essai, et en faisant varier différents paramètres tels que l'amortissement retrouver par simulation un tel phénomène de cascade harmonique. Antérieurement, au cours d'une autre étude [24], nous avions pu identifier ce type de cascade, mais il s'agissait alors de correction parabolique courte. Par contre, on notera que les résonances sous harmoniques sont elles assez bien respectées en terme de niveau relativement les unes par rapport aux autres.

\section{Discussion et perspectives}

Dans cette étude, nous avons souhaité confronter un modèle d'engrènement original à des essais expérimentaux. L'originalité du modèle repose dans le fait que la raideur d'engrènement est évaluée de manière instantanée à partir de la réponse temporelle, contrairement à la plupart des modèles existants. En effet, nous avions pu déjà mettre en évidence une plus grande richesse de phénomènes pour ce modèle dans une étude précédente [24]. Toutefois, les résultats comparatifs présentés dans cette étude n'apparaissent pas pleinement satisfaisants, et notamment au niveau de la description de la résonance principale. En perspective à ces travaux, nous sommes en train d'étudier l'effet de plusieurs paramètres susceptibles d'améliorer la description du phénomène non-linéaire. Plus précisément, il est certainement important d'améliorer le modèle en prenant en compte de manière plus réaliste les sources dissipatives qui jouent un rôle essentiel dans les phénomènes de résonance. Par ailleurs, nous avons entrepris également de prendre en compte d'une part la non-linéarité de la raideur d'engrènement associée à un seul couple de dents en prise et qui résulte entre autres de la présence d'un bombé longitudinal $(5 \mu \mathrm{m})$ sur la denture non négligeable vis-àvis de la correction de denture $(4 \mu \mathrm{m})$, et d'autre part, de l'effet d'une raideur mise en série à la raideur nonlinéaire d'engrènement développée dans cette étude. Ce dernier point peut se justifier par le fait que l'élasticité de la jante et de l'arbre pour des mouvements de torsion peut s'avérer d'un même ordre de grandeur que la raideur d'engrènement. Ces extensions font l'objet d'une étude en cours.

\section{Références}

[1] L.S. Harris, Dynamic loads on the teeth of spur gears, I. Mech. E. 172 (1958) 87-112

[2] R.W. Gregory, S.L. Harris, R.G. Munro, Dynamic behavior of spur gears, I. Mech. E. 178 (1964) 207-226

[3] G. Niemann, J. Baethge, Drehwegfehler, zahnfedernärte und gerdush bei stirnrädern, VDI-Z 112 (1970) 205-214

[4] G. Niemann, J. Baethge, Drehwegfehler, Zahnfedernärleund gerdush bei stirnrädern, VDI-Z 112 (1970) 475-499

[5] D.B. Welbourn, Fundamental knowledge of gear noise a survey, Proceedings of the Conference on Noise and Vibration of Engines and Transmissions, I. Mech. E. C117/79 (1979) 9-14

[6] H.N. Özgüven, D.R. Houser, Dynamic analysis of high speed gears by using loaded static transmission error, J. Sound Vib. 125 (1988) 71-83

[7] A. Kahraman, R. Singh, Non-linear dynamics of a spur gear, J. Sound Vib. 142 (1990) 49-75 
[8] R. Kasuba, J.W. Evans, An extended model for determining dynamic loads in spur gearing, ASME J. Mech. Design 103 (1981) 398-409

[9] F. Kücükay, Dynamic behaviour of high speed gears, I. Mech. E. C317/84 (1984) 81-90

[10] D.C.H. Yang, J.Y. Lin, Hertzian damping, tooth friction and bending elasticity in gear impact dynamics, ASME J. Mechanisms, Transmissions and Automation in Design 109 (1987) 189-196

[11] H.-H. Lin, R.L. Huston, J.J. Coy, On dynamic loads in parallel shaft transmissions: part I - Modelling and analysis, ASME J. Mechanisms, Transmissions and Automation in Design 110 (1988) 221-225

[12] A. Kahraman, R. Singh, Interactions between timevarying mesh stiffness and clearance non-linearities in a geared system, J. Sound Vib. 146 (1991) 135-156

[13] G.W. Blankenship, A. Kahraman, Steady state forced response of a mechanical oscillator with combined parametric excitation and clearance type non-linearity, J. Sound Vib. 185 (1995) 743-765

[14] A. Kahraman, G.W. Blankenship, Interactions between commensurate parametric and forcing excitations in a system with clearance, J. Sound Vib. 194 (1996) 317-336

[15] K.Y. Yoon, S.S. Rao, Dynamic load analysis of spur gears using a new tooth profile, ASME J. Mech. Design 118 (1996) $1-6$

[16] S. Theodossiades, S. Natsavias, Non-linear dynamics of gear-pair systems with periodic stiffness and backlash, J. Sound Vib. 229 (2000) 287-310

[17] K. Ichimaru, F. Hirano, Dynamic behavior of heavyloaded spur gears, ASME J. Eng. Industry 96 (1974) 373381
[18] R.W. Cornell, W.W. Westervelt, Dynamic tooth loads and stressing for high contact ratio spur gears, ASME J. Mech. Design 100 (1978) 69-76

[19] K.L. Wang, H.S. Cheng, A numerical solution to the dynamic load, film thickness, and surface temperatures in spur gears, part I Analysis, ASME J. Mech. Design 103 (1981) 177-187

[20] J. Perret-Liaudet, J. Sabot, On some bifurcations in a single-degree-of freedom gear model with backlash and mesh stiffness non-linearities, 4th World Congress on Gearing and Power Transmission, Paris 3, 1999, pp. 20032015

[21] A. Kahraman, G.W. Blankenship, Experiments on nonlinear dynamic behavior of an oscillator with clearance and periodically time-varying parameters, ASME J. Appl. Mech. 64 (1997) 217-226

[22] A. Kahraman, G.W. Blankenship, Effect of involute contact ratio on spur gear dynamics, ASME J. Mech. Design 121 (1999) 112-118

[23] A. Kahraman, G.W. Blankenship, Effect of involute tip relief on dynamic response of spur gear pairs, ASME J. Mech. Design 121 (1999) 313-315

[24] J. Perret-Liaudet, J. Sabot, Sur quelques comportements dynamiques non-linéaires des engrenages, Mécanique Industrielle et Matériaux 51 (1998) 170-172

[25] A. Nayfeh, B. Balachandran, Applied Nonlinear Dynamics, John Wiley and Sons, New York, 1995

[26] R. Seydel, Practical Bifurcation and Stability Analysis From Equilibrium to Chaos, Springer, Berlin, 1994 